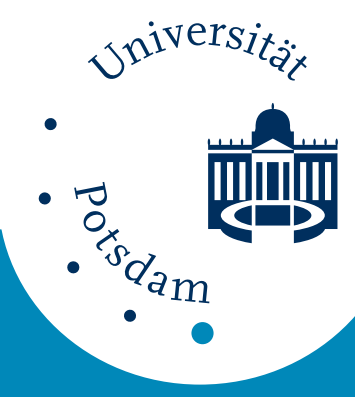

Universität Potsda m

Stefan Scheel, Norbert Seehafer

Bifurcation to oscillations in three-dimensional Rayleigh-Bénard convection

NLD Preprints ; 39 


\title{
Bifurcation to oscillations in three-dimensional Rayleigh-Bénard convection
}

\author{
S. Scheel and N. Seehafer \\ Institut für Physik, Universität Potsdam, PF 601553, D-14415 Potsdam, Germany
}

(August 21, 1997)

\begin{abstract}
Three-dimensional bouyancy-driven convection in a horizontal fluid layer with stress-free boundary conditions at top and bottom and periodic boundary conditions in the horizontal directions is investigated by means of numerical simulation and bifurcation-analysis techniques. The aspect ratio is fixed to a value of $2 \sqrt{2}$ and the Prandtl number to a value of 6.8 . Two-dimensional convection rolls are found to be stable up to a Rayleigh number of 17950 , where a Hopf bifurcation leads to travelling waves. 'I'hese are stable up to a Rayleigh number of 30000, where a secondary Hopf bifurcation generates modulated traveling waves. We pay particular attention to the symmetries of the solutions and symmetry breaking by the bifurcations.
\end{abstract}

47.20.Ky, 47.20.Bp, 47.54.+r

\section{INTRODUCTION}

Rayleigh-Bénard convection, that is, buoyancy-driven convection in a fluid layer heated from below and cooled from above, is one of the prime examples of bifurcating high-dimensional systems. It has long since been a subject of intense theoretical and experimental study [1-4]. Important applications are e.g. in meteorology, geophysics and astrophysics.

From the theoretical side, several approaches have been used to analyze and understand the dynamics of the Rayleigh-Bénard system, in particular the formation of the different stationary or time-dependent patterns observed in experiments, as well as the steps in the evolution towards turbulence. On one hand there are calculations of equilibria and instabilities using perturbationtheoretic methods [3,5-8]. Most current theoretical knowledge on convective patterns seems to have been obtained by this kind of analysis. More recently, also methods of bifurcation theory $[9,10]$ are applied, by which one, e.g., derives low-dimensional systems of amplitude equations describing the qualitative behavior of the system close to a bifurcation point, thus allowing for the characterization of the bifurcation. If symmetries are present, as is the case for the Rayleigh-Bénard system, grouptheoretic methods are an important tool of the theoretical bifurcation analysis $[11,12]$.

On the other hand, there are numerical simulations in which the governing partial differential equations are integrated forward in time, starting from selected or random initial conditions $[13,14]$. To some extent the simulations can allow also a bifurcation analysis, namely, to get an overview of the possible time-asymptotic states for given values of the system parameters. The bifurcation analysis is facilitated if additionally more specific numerical methods are used, like eigenvalue calculations at given equilibrium states (which are given only numerically) and the direct tracing of solution branches.

In general, convection sets in in the form of stationary patterns, notably two-dimensional straight rolls, which for stronger buoyancy forces then lose their stability to time-dependent convection. Small Prandtl numbers of the fluid are favorable for an early transition to time dependence. Different oscillatory instabilities of convection rolls were studied by Busse [15], Clever and Busse [16,17], and by Bolton et al. [18]. In Ref. [17] also the bifurcating new time-asymptotic, oscillatory state was calculated, namely, waves propagating along the roll axis.

In convection experiments various oscillations are observed. Their character and, in particular, the kind of the transition from stationary to nonstationary convection is distinctly different for small and large aspect ratios $L$ (widths of the fluid layers in units of their depths); relevant experiments are summarized, e.g., by Behringer [19] and by Koschmieder [4]. For small aspect ratios ( $L$ less than about 10) only a few sharp frequencies are present at the onset of time dependence, while for large aspect ratios a broadband spectrum appears and the transition is more gradual. In numerical simulations addressing the latter situation for a low Prandtl number fluid, Xi et al. [20] recently found spatiotemporal chaos immediately after the onset of convection.

In this paper the small aspect ratio situation is considered. We use numerical methods to study, for an aspect ratio of $2 \sqrt{2}$ and a Prandtl number of 6.8 (corresponding to water at room temperature), the first three bifurcations in three-dimensional Rayleigh-Bénard convection with stress-free boundary conditions at top and bottom. Particular attention is paid to symmetry and symmetry breaking.

After introducing the governing equations in Sec. II, we describe appearance and stability of two-dimensional convection rolls in Sec. III, followed by a discussion of their symmetries in Sec. IV. Then, in Sec. V, we study bifurcations of the rolls to periodic and quasiperiodic oscillations. Sec. VI gives a discussion of the results.

\section{EQUATIONS}

We consider buoyancy-driven convection in a plane fluid layer of thickness $d$ heated from below. Using the 
Oberbeck-Boussinesq approximation, the governing system of partial differential equations reads as follows:

$$
\begin{aligned}
\frac{\partial \mathbf{v}}{\partial t}+(\mathbf{v} \cdot \nabla) \mathbf{v} & =-\nabla p+\mathcal{P} \triangle \mathbf{v}+\mathcal{P} \mathcal{R} \theta \mathbf{e}_{z} \\
\nabla \cdot \mathbf{v} & =0 \\
\frac{\partial \theta}{\partial t}+\mathbf{v} \cdot \nabla \theta & =v_{z}+\triangle \theta .
\end{aligned}
$$

Here $\mathbf{v}$ is the fluid velocity and $p$ and $\theta$ represent the deviations of pressure and temperature from their values in the pure conduction state. We use Cartesian coordinates $x, y$ and $z$ with the $z$ axis in the vertical direction parallel to the gravitational force. $\mathbf{e}_{z}$ is the unit vector in the vertical direction. Equations (1)-(3) are given in dimensionless form where the units of length and time are $d$ and $\kappa / d^{2}$, respectively, with $\kappa$ being the thermal diffusivity. $\theta$ is measured in units of the temperature difference $\delta T$ between lower and upper boundaries of the fluid layer. There are two dimensionless parameters, the Prandtl number $\mathcal{P}$ and the Rayleigh number $\mathcal{R}$, defined by

$$
\mathcal{P}=\frac{\nu}{\kappa}, \quad \mathcal{R}=\frac{\alpha g d^{3}}{\nu \kappa} \delta T,
$$

where $\nu$ is the kinematic viscosity, $\alpha$ the volumetric expansion coefficient and $g$ the gravitational acceleration. The Rayleigh number $\mathcal{R}$ measures the strength of the buoyancy forces.

We apply periodic boundary conditions with spatial period $L$ in the horizontal directions $x$ and $y$. The top and bottom planes are assumed to be impenetrable, stress-free and isothermal:

$$
\frac{\partial v_{x}}{\partial z}=\frac{\partial v_{y}}{\partial z}=v_{z}=\theta=0 \quad \text { at } z=0,1 .
$$

For these boundary conditions the following Fourier expansions are appropriate:

$$
\begin{aligned}
& v_{x}=\sum_{k_{x}, k_{y}=-\infty}^{\infty} \sum_{k_{z}=0}^{\infty} \tilde{v}_{x}(\mathbf{k}) \mathrm{e}^{\mathrm{i} k_{x} x+\mathrm{i} k_{y} y} \cos k_{z} z \\
& v_{y}=\sum_{k_{x}, k_{y}=-\infty}^{\infty} \sum_{k_{z}=0}^{\infty} \tilde{v}_{y}(\mathbf{k}) \mathrm{e}^{\mathrm{i} k_{x} x+\mathrm{i} k_{y} y} \cos k_{z} z \\
& v_{z}=\sum_{k_{x}, k_{y}=-\infty}^{\infty} \sum_{k_{z}=0}^{\infty} \tilde{v}_{z}(\mathbf{k}) \mathrm{e}^{\mathrm{i} k_{x} x+\mathrm{i} k_{y} y} \sin k_{z} z \\
& \theta=\sum_{k_{x}, k_{y}=-\infty}^{\infty} \sum_{k_{z}=0}^{\infty} \tilde{\theta}(\mathbf{k}) \mathrm{e}^{\mathrm{i} k_{x} x+\mathrm{i} k_{y} y} \sin k_{z} z \\
& p=\sum_{k_{x}, k_{y}=-\infty}^{\infty} \sum_{k_{z}=0}^{\infty} \tilde{p}(\mathbf{k}) \mathrm{e}^{\mathrm{i} k_{x} x+\mathrm{i} k_{y} y} \cos k_{z} z
\end{aligned}
$$

The wavenumbers $\mathbf{k}=\left(k_{x}, k_{y}, k_{z}\right)$ are connected with the integer modenumbers $\mathbf{n}=\left(n_{x}, n_{y}, n_{z}\right)$ by

$$
\begin{array}{rlrl}
k_{x} & =n_{x} \frac{2 \pi}{L}, & & n_{x}=0, \pm 1, \pm 2, \ldots \\
k_{y}=n_{y} \frac{2 \pi}{L}, & n_{y}=0, \pm 1, \pm 2, \ldots \\
k_{z}=n_{z} \pi, & n_{z}=0,1,2,3, \ldots
\end{array}
$$

With the abbreviations

$$
\mathbf{w}=(\mathbf{v} \cdot \nabla) \mathbf{v}, \quad \phi=\mathbf{v} \cdot \nabla \theta
$$

for the nonlinearities, which have Fourier expansions like $\mathbf{v}$ and $\theta$, we arrive at the analogues of Eqs. (1)-(3) in Fourier space:

$$
\begin{aligned}
0 & =\mathrm{i} k_{x} \tilde{v}_{x}+\mathrm{i} k_{y} \tilde{v}_{y}+k_{z} \tilde{v}_{z} \\
\dot{\tilde{v}}_{x} & =-\mathrm{i} k_{x} \tilde{p}-\tilde{w}_{x}-\mathcal{P} \mathbf{k}^{2} \tilde{v}_{x} \\
\dot{\tilde{v}}_{y} & =-\mathrm{i} k_{y} \tilde{p}-\tilde{w}_{y}-\mathcal{P} \mathbf{k}^{2} \tilde{v}_{y} \\
\dot{\tilde{v}}_{z} & =k_{z} \tilde{p}-\tilde{w}_{z}-\mathcal{P} \mathbf{k}^{2} \tilde{v}_{z}+\mathcal{P} \mathcal{R} \tilde{\theta} \\
\dot{\tilde{\theta}} & =\tilde{v}_{z}-\tilde{\phi}-\mathbf{k}^{2} \tilde{\theta} .
\end{aligned}
$$

Due to the constraint (2) not all of these equations are independent of each other. By taking the divergence of Eq. (1) one eliminates the pressure $p$, namely,

$$
\tilde{p}=\mathrm{i} \frac{k_{x}}{\mathbf{k}^{2}} \tilde{w}_{x}+\mathrm{i} \frac{k_{y}}{\mathbf{k}^{2}} \tilde{w}_{y}+\frac{k_{z}}{\mathbf{k}^{2}} \tilde{w}_{z}-\frac{k_{z}}{\mathbf{k}^{2}} \tilde{\theta}
$$

Equations (16) and (17) can now be written as

$$
\begin{aligned}
\dot{\tilde{v}}_{x}= & \left(\frac{k_{x}^{2}}{\mathbf{k}^{2}}-1\right) \tilde{w}_{x}+\frac{k_{x} k_{y}}{\mathbf{k}^{2}} \tilde{w}_{y}-\mathcal{P} \mathbf{k}^{2} \tilde{v}_{x} \\
& +\mathrm{i} \frac{k_{x} k_{z}}{\mathbf{k}^{2}}\left(\mathcal{P} \mathcal{R} \tilde{\theta}-\tilde{w}_{z}\right) \\
\dot{\tilde{v}}_{y}= & \left(\frac{k_{y}^{2}}{\mathbf{k}^{2}}-1\right) \tilde{w}_{y}+\frac{k_{x} k_{y}}{\mathbf{k}^{2}} \tilde{w}_{x}-\mathcal{P} \mathbf{k}^{2} \tilde{v}_{y} \\
& +\mathrm{i} \frac{k_{y} k_{z}}{\mathbf{k}^{2}}\left(\mathcal{P} \mathcal{R} \tilde{\theta}-\tilde{w}_{z}\right) .
\end{aligned}
$$

We restrict ourselves to the case of a vanishing mean horizontal flow, that is $\tilde{v}_{x}(0,0,0)=\tilde{v}_{y}(0,0,0)=0$, since any such flow can be removed by a Galilean transformation. This can be seen from the relations

$$
\begin{aligned}
& \mathbf{v} \cdot \nabla v_{x}=\frac{\partial}{\partial x} v_{x}^{2}+\frac{\partial}{\partial y} v_{x} v_{y}+\frac{\partial}{\partial z} v_{x} v_{z} \\
& \mathbf{v} \cdot \nabla v_{y}=\frac{\partial}{\partial y} v_{y}^{2}+\frac{\partial}{\partial x} v_{x} v_{y}+\frac{\partial}{\partial z} v_{y} v_{z}
\end{aligned}
$$

which show, together with Eqs. (14), (16) and (17) and the boundary conditions, that $\tilde{v}_{x}(0,0,0)$ and $\tilde{v}_{y}(0,0,0)$ are time-independent.

\section{TWO-DIMENSIONAL STABLE ROLLS}

For the numerical calculations we used a pseudospectral code $[21,22]$ with 16 collocation points in each spatial direction. Time integration was performed using an 
eighth-order Runge-Kutta scheme with adaptive timestepping according to [23]. The steady-state solutions were reached after $0.5 \ldots 1$ in our time units which corresponds to one or two hours computation time on a DECalpha machine with $150 \mathrm{MHz}$. However, to get onto an attractor for time-dependent states increases the computation time considerably.

In an infinitely extended fluid layer (with no lateral boundaries), the critical value of $\mathcal{R}$ for the transition from the trivial ground state $\mathbf{v}=\theta=0$ (the pure conduction state) to convection can be calculated analytically and gives $\mathcal{R}_{c}=657.5$ independent of the Prandtl number of the fluid $[1,2,4,11]$. The associated unstable modes are nonoscillatory, with a vertical modenumber of $n_{z}=1$ and a horizontal wavelength of $2 \sqrt{2}$ - chosen to be our aspect ratio $L$. Correspondingly we find the modes with the lowest modenumbers, namely the modes $( \pm 1,0,1)$, to become unstable first.

The unstable perturbations do not yet determine the bifurcating new time-asymptotic state. By applying group-theoretical methods, it has been shown that steady-state bifurcations from the conductive state can lead to convection in the form of two-dimensional rolls, hexagons or regular triangles $[11,24]$. For our parameters we find purely two-dimensional, straight, stationary convection rolls, in accordance with what is ordinarily seen in experiments in closed containers [4]. With the roll axes in the $y$ direction (an orientation in the $x$ direction is equally possible), there is then a number of symmetry relations for the modes $(i, 0, k)$ (see Sec. IV). There is no $y$ dependence and no velocity component in the $y$ direction. Velocity streamlines in the $x-z$ plane are shown in Fig. 1.

The two-dimensional stationary roll solution remains stable up to a second critical Rayleigh number at $\mathcal{R}=$ $17950=27.3 \mathcal{R}_{c}$. At this point a Hopf bifurcation is observed (cf. Sec. V).

Now according to analytical and numerical threedimensional stability studies, two-dimensional convection rolls are subject to various kinds of instabilities, notably the zig-zag, cross-roll, and skewed varicose instabilities, as treated for stress-free boundaries in Refs. [7,8] (see also the discussion in Ref. [3], though this primarily refers to rigid boundaries). These instabilities do not seem to be relevant for our special case, however. They are thought to have the purpose to decrease or increase the wavelength of the convection rolls. For $\mathcal{P} \approx 7$, they affect only roll solutions with wavelengths larger than the critical one (our $L$ ). As we have fixed our aspect ratio $L$ to the critical wavelength, it is then not surprising that we do not see these instabilities. Furthermore, the instabilities mentioned (the unstable perturbations) are mainly longwave (with wavelengths larger than $L$ ) and are thus not admitted by our periodic boundary conditions. Differences may also arise from the fact that we do not allow a continuum of wavelengths for the perturbations. So on the side of the small wavelengths, the one closest to $L$ is $L / 2$. This reflects the situation of bounded physical systems.

\section{SYMMETRIES}

At the onset of convection, when the bifurcation parameter $\mathcal{R}$ exceeds its critical value only slightly, the resulting pattern is symmetric with respect to the following discrete and continuous symmetries:

$$
\begin{aligned}
S_{1}:(x, y, z) & \mapsto(-x, y, z) \\
\left(v_{x}, v_{y}, v_{z}, \theta\right) & \mapsto\left(-v_{x}, v_{y}, v_{z}, \theta\right) \\
S_{2}: \quad(x, y, z) & \mapsto\left(x+\frac{L}{2}, y, 1-z\right) \\
\left(v_{x}, v_{y}, v_{z}, \theta\right) & \mapsto\left(v_{x}, v_{y},-v_{z},-\theta\right) \\
S_{3}: \quad(x, y, z) & \mapsto(x,-y, z) \\
\left(v_{x}, v_{y}, v_{z}, \theta\right) & \mapsto\left(v_{x},-v_{y}, v_{z}, \theta\right) \\
T(a): \quad(x, y, z) & \mapsto(x, y+a, z) \\
\left(v_{x}, v_{y}, v_{z}, \theta\right) & \mapsto\left(v_{x}, v_{y}, v_{z}, \theta\right)
\end{aligned}
$$

Here $S_{1}$ is a reflection in the $y$ - $z$ plane or the vertical midplane separating rolls, $S_{2}$ a reflection in the $x$ - $y$ plane or the horizontal midplane, combined with a translation of the pattern in the $x$ direction by one roll or $L / 2$, and $S_{3}$ a reflection in a plane cutting the rolls alongside. The only continuous group operation, $T(a)$, is a translation along the roll axis (the $y$ direction) by $a, a \in[0, L], a \in \mathbb{R}$.

There are also symmetries with respect to products of the basic discrete group elements, namely to $S_{4}=S_{1} \circ S_{2}$, $S_{5}=S_{2} \circ S_{3}$ and $S_{6}=S_{1} \circ S_{3}$ with

$$
\begin{aligned}
S_{4}:(x, y, z) & \mapsto\left(-x+\frac{L}{2}, y, 1-z\right) \\
\left(v_{x}, v_{y}, v_{z}, \theta\right) & \mapsto\left(-v_{x}, v_{y},-v_{z},-\theta\right) \\
S_{5}: \quad(x, y, z) & \mapsto\left(x+\frac{L}{2},-y, 1-z\right) \\
\left(v_{x}, v_{y}, v_{z}, \theta\right) & \mapsto\left(v_{x},-v_{y},-v_{z},-\theta\right) \\
S_{6}: \quad(x, y, z) & \mapsto(-x,-y, z) \\
\left(v_{x}, v_{y}, v_{z}, \theta\right) & \mapsto\left(-v_{x},-v_{y}, v_{z}, \theta\right)
\end{aligned}
$$

as well as symmetries with respect to the products between the discrete elements and the continuous element $T(a)$.

The symmetry group $\mathcal{G}=\left\{i d, S_{1}, \ldots, S_{6}, T\right\}$, which consists of all these operations, is abelian. Each of the discrete elements is inverse to itself.

The different symmetries have their specific consequences for the Fourier coefficients of the velocity and 
temperature fields. For example, symmetry to $S_{1}$ requires

$$
\begin{aligned}
\tilde{v}_{x}(i, j, k) & =-\tilde{v}_{x}(-i, j, k) \\
\tilde{v}_{y}(i, j, k) & =\tilde{v}_{y}(-i, j, k) \\
\tilde{v}_{z}(i, j, k) & =\tilde{v}_{z}(-i, j, k) \\
\tilde{\theta}(i, j, k) & =\tilde{\theta}(-i, j, k),
\end{aligned}
$$

for a solution symmetric with respect to $S_{2}$ one has

$$
\begin{aligned}
\tilde{v}_{x}(i, j, k)=\tilde{v}_{y}(i, j, k)=\tilde{v}_{z}(i, j, k)= & \tilde{\theta}(i, j, k)=0 \\
& i+k \text { odd }
\end{aligned}
$$

while for symmetry to $S_{4}$ the following relations hold:

$$
\begin{aligned}
\tilde{v}_{x}(i, j, k) & =(-1)^{i+k+1} \tilde{v}_{x}(-i, j, k) \\
\tilde{v}_{y}(i, j, k) & =(-1)^{i+k} \tilde{v}_{y}(-i, j, k) \\
\tilde{v}_{z}(i, j, k) & =(-1)^{i+k} \tilde{v}_{z}(-i, j, k) \\
\tilde{\theta}(i, j, k) & =(-1)^{i+k} \tilde{\theta}(-i, j, k)
\end{aligned}
$$

The translational symmetry of the system in the $x$ direction leads to a continuum of coexisting solutions. Only for one particular among them the symmetries $S_{1}$ and $S_{4}$ are correctly expressed by Eqs. (32) and (34); otherwise still phase factors corresponding to $x$ translations appear.

The subgroup formed by the transformations $S_{1}, S_{2}$, $S_{4}$, and the identity is also found in studies of twodimensional convection $[25,26]$. It is isomorphic to the dihedral group $D_{2}$ [11]. The formal structure of the total group $\mathcal{G}$ is then $D_{2} \times O(2)$.

\section{BIFURCATIONS TO PERIODIC AND QUASIPERIODIC SOLUTIONS}

The second bifurcation at $\mathcal{R}=17950=27.3 \mathcal{R}_{c}$ is a Hopf bifurcation in which two identical pairs of complex conjugate eigenvalues pass the imaginary axis. It leads to traveling waves propagating along the roll axis, i.e. in the positive or negative $y$ direction. The multiplicity of the eigenvalues is obviously due to the $O(2)$ symmetry with respect to this direction. It is desribed e.g. in the review by Crawford and Knobloch [12] that in nondegenerate Hopf bifurcations under $O(2)$ symmetry standing and traveling waves bifurcate simultaneously, that a stable new solution exists only if both bifurcate supercritically, and that at most one of them can be stable (which one is selected is then determined by the parameters of the problem). Traveling waves have been found for the case of no-slip boundary conditions by Clever and Busse [17].

At the bifurcation point the time period of the oscillations, determined from the imaginary part of the critical eigenvalues, is $\tau=0.066$ in our time units (the thermal diffusion time). At $\mathcal{R}=20000$ we measured $\tau=0.064$. The period of the modes $(i, j, k)$ is $\tau / j$ if $j \neq 0$. The modes $(i, 0, k)$ are still time-independent. There is now a nonvanishing flow component, $v_{y}$, in the axial direction. Snapshots of the velocity profiles at $t=0, t=\tau / 4, t=\tau / 2$ and $t=3 \tau / 4$ are shown in Fig. 2 .

The critical $\mathcal{R}$ for the Hopf bifurcation as well as the time period of the oscillations may be compared with Busse's theory [15] for the instability of convection rolls to perturbations in the form of traveling waves under stress-free boundary conditions. According to the theory, the critical $\mathcal{R}$ satisfies $\mathcal{R} / \mathcal{R}_{c}=0.31 \mathcal{P}^{2}+1$, which for our parameters would give $\mathcal{R} / \mathcal{R}_{c}=15.3$, i.e. a significantly smaller Rayleigh number than we have found. This is again explained by the suppression of long-wave perturbations, which would become unstable earlier. For the time period of the oscillations Busse gives [Eq. (5.35b) in Ref. [3]] $\tau=\left[2 \mathcal{R}_{c} / 3\left(\mathcal{R}-\mathcal{R}_{c}\right) b^{2}\right]^{1 / 2}$, where $b$ is the (axial) wavenumber of the waves. The dependence of $\tau$ on $b$ contained in this formula admits a direct comparison with the periods found in our numerical calculations. According to the formula, $\tau=0.0717$ at our Hopf bifurcation point (with $b=\pi / \sqrt{2}$ ), which is in good agreement with the value of 0.066 from the purely numerical calculation. Also for Rayleigh numbers above the Hopf value, the comparison of the time periods gives satisfactory results, but there it is no longer really appropriate as the periods from the formula refer to unstable perturbation to the stationary convection rolls and not to solutions on the new branch, which will depart more and more from the (unstable) roll branch.

The Hopf bifurcation breaks the $O(2)$ symmetry in the $y$ direction. More precisely, the reflection symmetry to $S_{3}$ is broken - the application of $S_{3}$ changes the direction of the wave propagation, while each periodic orbit as a whole is still invariant with respect to translations $T$, which merely produce a time shift. If we formally characterize the $y-t$ symmetry before the bifurcation by $O(2)_{y} \times S O(2)_{t}$, where $S O(2)_{t}$ is the circular symmetry with respect to time, the remaining symmetry after the bifurcations is $S O(2)_{y-c t}$, with $c$ denoting the velocity of the wave propagation.

An inspection of the Fourier coefficients shows that also the symmetries $S_{1}$ and $S_{4}$ are broken but that the following still holds (after, if necessary, the solution is appropriately shifted in the $x$ direction):

$$
\begin{aligned}
\tilde{v}_{x}(i, j, k) & =0 \quad i+k \text { odd } \\
\tilde{v}_{y}(i, j, k) & =0 \quad i+k \text { odd } \\
\tilde{v}_{z}(i, j, k) & =0 \quad i+k \text { odd } \\
\tilde{\theta}(i, j, k) & =0 \quad i+k \text { odd } \\
\tilde{v}_{x}(-i, j, k) & =(-1)^{j+1} \tilde{v}_{x}(i, j, k) \\
\tilde{v}_{y}(-i, j, k) & =(-1)^{j} \tilde{v}_{y}(i, j, k) \\
\tilde{v}_{z}(-i, j, k) & =(-1)^{j} \tilde{v}_{z}(i, j, k) \\
\tilde{\theta}(-i, j, k) & =(-1)^{j} \tilde{\theta}(i, j, k)
\end{aligned}
$$

These relations show that the symmetry $S_{2}$ is retained in its original form and that the solutions are sym- 
metric with respect to $S_{1} \circ T(L / 2)$, i.e. a left-right reflection followed by an $L / 2$ translation in the $y$ direction. This implies that there is also a symmetry to $S_{2} \circ S_{1} \circ T(L / 2)=S_{4} \circ T(L / 2)$. The symmetry group $\mathcal{G}$ of the stationary convection rolls may, differently from the choice in Sec. IV, also be generated by the four transformations $S_{1} \circ T(L / 2), S_{2}, S_{3}$, and $T$. Of the three discrete symmetries here then just one, that one corresponding to $S_{3}$, is broken in the Hopf bifurcation. The remaining spatial symmetry group (time shifts not taken into account) is generated by $S_{1} \circ T(L / 2)$ and $S_{2}$ and consists of the elements $S_{1} \circ T(L / 2), S_{2}, S_{4} \circ T(L / 2)$, and the identity. It is isomorphic to the dihedral group $D_{2}$.

On further increasing the Rayleigh number $\mathcal{R}$, at $\mathcal{R} \approx$ $30000=45.6 \mathcal{R}_{c}$ a third bifurcation is observed, which is again a Hopf bifurcation. The appearance of a second basic frequency (see Fig. 3) leads to a torus solution in phase space (depicted in Fig. 4). In physical space the solution can be characterized as a modulated traveling wave. Also the special modes $(i, 0, k)$ are now time-dependent, but they are simply periodic, oscillating with just one frequency, namely that of the modulation. There is now a periodic motion of the rolls not only in the $x$ direction but also in the $z$ direction.

For the motion being already quite complicated, the number of modes (16 collocation points in each spatial direction) is still sufficient. A closer look at modes, e.g. $(i, 1,0)$, with increasing $i$ reveals that their energy content decreases from $\mathcal{O}\left(10^{2}\right)$ in some suitable units for the modes $(0,1,0)$ to $\mathcal{O}\left(10^{-5}\right)$ for the modes $( \pm 5,1,0)$. We also performed test runs with 32 collocation points in each spatial direction, but since the results did not change and no other modes were excited we found it not worth increasing the CPU time by a factor of 8 .

In numerical simulations for comparable parameters ( $L=2 \sqrt{2}, \mathcal{P}=10$ ), as well as stress-free boundary conditions, Curry et al. [13] observed a transition from singlefrequency oscillations to a two-frequency quasiperiodic state at about the same Rayleigh number as we do.

The torus bifurcation is connected with a further symmetry breaking. In such a case the new solution can be symmetric to at most one of the three transformations of the symmetry group of the periodic solution. Actually the symmetry to $S_{1} \circ T(L / 2)$ survives while the other two symmetries are broken. So the $D_{2}$ symmetry of the periodic solution is broken to a $Z_{2}$ symmetry of the quasiperiodic solution.

\section{DISCUSSION}

We have studied Rayleigh-Bénard convection in a plane fluid layer with stress-free boundary conditions, using an aspect ratio $L$ of $2 \sqrt{2}$ and a Prandtl number $\mathcal{P}$ of 6.8. Two-dimensional convection rolls remain then stable up to a second critical Rayleigh number $\mathcal{R}=17950=27.3 \mathcal{R}_{c}$. Instabilities like the zig-zag, cross- roll, and skewed varicose instabilities seem to be prohibited by the choice of the aspect ratio and the associated suppression of long-wave instabilities.

The bifurcation at $\mathcal{R}=17950$ is a Hopf bifurcation leading to traveling waves along the roll axis. The resulting structures are wavy rolls, as calculated for the case of no-slip boundary conditions and Prandtl numbers up to 0.71 by Clever and Busse [17]. The frequencies we find agree very well with the theory of Busse [15], who in turn found them in good agreement with experiments, e.g. those of Krishnamurti [27] and others, with large aspect ratio containers. Compared to Busse's theory, we observe the waves to occur at higher Rayleigh numbers. This is again explained by the suppression of long-wave perturbations. The agreement with the experiments summarized in Ref. [27] is even improved by the shift to higher Rayleigh numbers, which must of course not be overestimated since, among other things, our aspect ratio is probably much too small to allow for a direct comparison with these experiments, to which Busse's theoretical approach appears to be better adapted. On the other hand, Gollub and Benson [28] report an experiment with $L=3.5, \mathcal{P}=5$ and a transition from stationary to simply-periodic convection at $\mathcal{R}=27.2 \tilde{\mathcal{R}}_{c}$, where $\tilde{\mathcal{R}}_{c}$ is the critical $\mathcal{R}$ for the onset of convection under no-slip boundary conditions.

The Hopf bifurcation breaks the spatial $D_{2} \times O(2)$ symmetry of the stationary convection rolls down to a $D_{2}$ symmetry. If symmetry with respect to time is included, the total symmetry is broken from $\left[D_{2}\right]_{x, z} \times O(2)_{y} \times$ $S O(2)_{t}$ to $\left[D_{2}\right]_{x, y, z} \times S O(2)_{y-c t}$.

At $\mathcal{R} \approx 30000=45.6 \mathcal{R}_{c}$ a secondary Hopf bifurcation leads to a torus solution in phase space. The corresponding pattern is a modulated traveling wave. In this bifurcation the spatial symmetry is broken from $D_{2}$ to $Z_{2}$ (which is now also the total symmetry). The modulated traveling waves are still symmetric with respect to leftright reflections followed by $L / 2$ translations in the (originally) axial direction.

The bifurcations to be expected for further increased Rayleigh numbers, which presumably lead to chaotic states, will be the subject of future studies.

\section{ACKNOWLEDGMENTS}

We wish to thank M. Zaks for helpful discussions.

[1] S. Chandrasekhar, Hydrodynamic and Hydromagnetic Stability (Clarendon Press, Oxford, 1961).

[2] T. G. Drazin and W. H. Reid, Hydrodynamic Stability (Cambridge University Press, Cambridge, England, 1981). 
[3] F. H. Busse, in Hydrodynamic Instabilities and the Transition to Turbulence, Vol. 45 of Topics in Applied Physics, edited by H. L. Swinney and J. P. Gollub (Springer, Berlin, Heidelberg, 1985), pp. 97-133.

[4] E. L. Koschmieder, Bénard Cells and Taylor Vortices (Cambridge University Press, Cambridge, 1993).

[5] A. Schlüter, D. Lortz, and F. H. Busse, J. Fluid Mech. 23, 129 (1965).

[6] F. H. Busse, Rep. Prog. Phys. 41, 1929 (1978).

[7] F. H. Busse and E. W. Bolton, J. Fluid Mech. 146, 115 (1984).

[8] E. W. Bolton and F. H. Busse, J. Fluid Mech. 150, 487 (1985).

[9] J. Guckenheimer and P. Holmes, Nonlinear Oscillations, Dynamical Systems, and Bifurcations of Vector Fields (Springer, New York, 1983).

[10] J. D. Crawford, Rev. Mod. Phys. 63, 991 (1991).

[11] M. Golubitsky, I. Stewart, and D. G. Schaeffer, Singularities and Groups in Bifurcation Theory (Springer, New York, 1988), Vol. II.

[12] J. D. Crawford and E. Knobloch, Annu. Rev. Fluid Mech. 23, 341 (1991).

[13] J. H. Curry, J. R. Herring, J. Loncaric, and S. A. Orszag, J. Fluid Mech. 147, 1 (1984).

[14] M. Meneguzzi, C. Sulem, P. L. Sulem, and O. Thual, J. Fluid Mech. 182, 169 (1987).

[15] F. H. Busse, J. Fluid Mech. 52, 97 (1972).

[16] R. M. Clever and F. H. Busse, J. Fluid Mech. 65, 625 (1974).

[17] R. M. Clever and F. H. Busse, J. Fluid Mech. 176, 403 (1987).

[18] E. W. Bolton, F. H. Busse, and R. M. Clever, J. Fluid Mech. 164, 469 (1986).

[19] R. P. Behringer, Rev. Mod. Phys. 57, 657 (1985).

[20] H. Xi, X. Li, and J. D. Gunton, Phys. Rev. Lett. 78, 1046 (1997).

[21] C. Canuto, M. Y. Hussaini, A. Quarteroni, and T. A. Zang, Spectral Methods in Fluid Dynamics (Springer, Berlin, 1988).

[22] W. H. Press, B. P. Flannery, S. A. Teukolsky, and W. T. Vetterling, Numerical Recipes, The Art of Scientific Computing, FORTRAN Version (Cambridge University Press, Cambridge, 1989).

[23] E. Hairer and G. Wanner, Solving Ordinary Differential Equations II (Springer, Berlin, 1991).

[24] M. Golubitsky, J. W. Swift, and E. Knobloch, Physica D 10, 249 (1984).

[25] J. Prat, J. M. Massaguer, and I. Mercader, Phys. Fluids 7, 121 (1995).

[26] E. Zienicke, N. Seehafer, and F. Feudel, Bifurcations in two-dimensional Rayleigh-Bénard convection, 1997, submitted to Phys. Rev. E.

[27] R. Krishnamurti, J. Fluid Mech. 42, 309 (1970).

[28] J. P. Gollub and S. V. Benson, J. Fluid Mech. 100, 449 (1980).

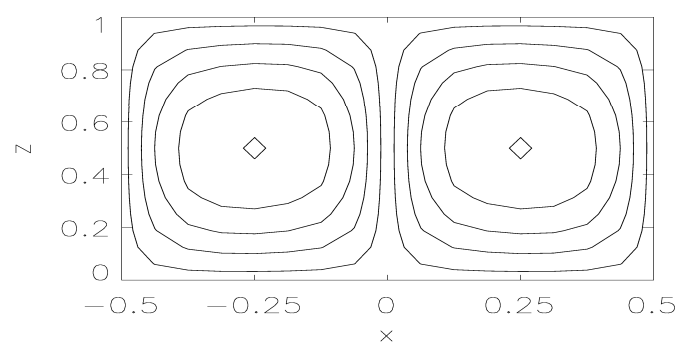

FIG. 1. Velocity streamlines at $\mathcal{R}=12000=18.25 \mathcal{R}_{c} . \quad x$ and $z$ are measured in units of $L$ and $d$, respectively.
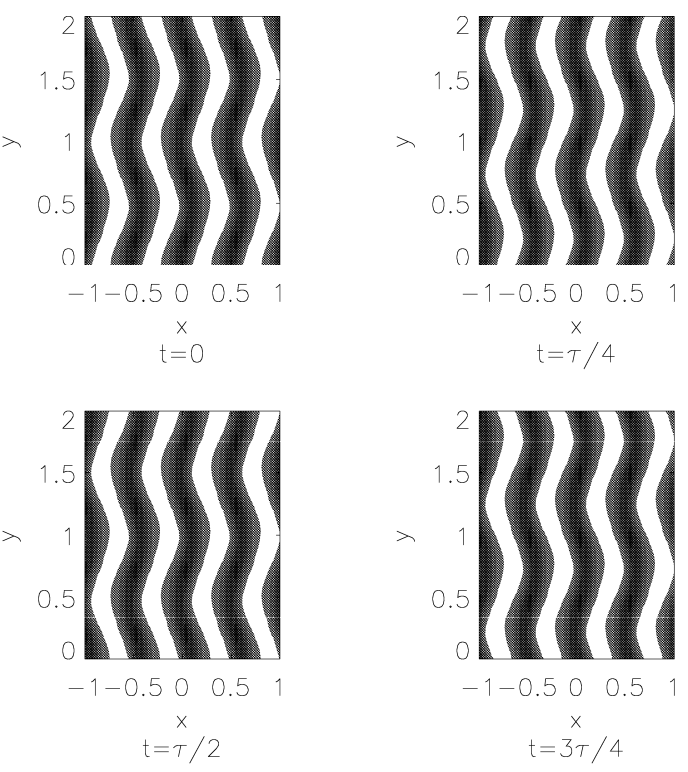

FIG. 2. Snapshots of the modulus of the velocity field in the horizontal midplane at different times, calculated for $\mathcal{R}=20000=30.4 \mathcal{R}_{c}$. Four neighboring periodic boxes are shown, two in each horizontal direction. $x$ and $y$ are measured in units of $L, \tau=0.066$ is the time period measured in units of the thermal diffusion time. 


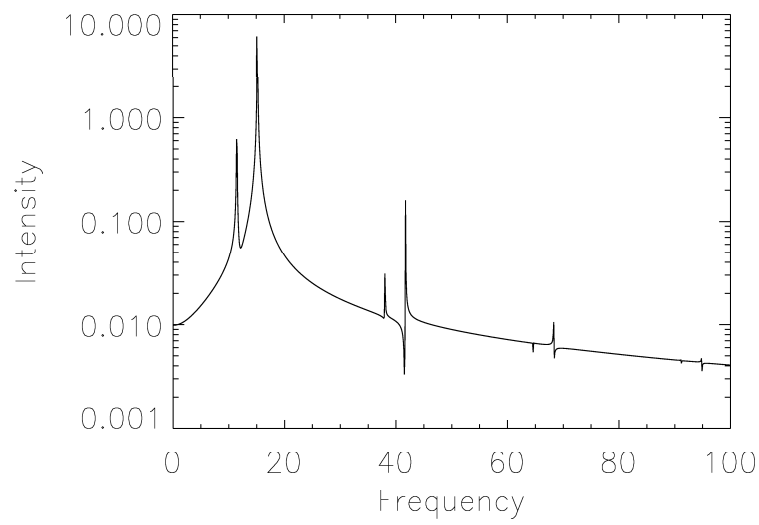

FIG. 3. Power spectrum of the temporal evolution of $\Re v_{y}(-1,1,1)$ at $\mathcal{R}=32000=48.7 \mathcal{R}_{c}$. Frequencies are measured in units of the inverse thermal diffusion time $\kappa / d^{2}$. The two basic frequencies are $f_{1}=15$ (traveling wave) and $f_{2}=3.5$ (modulation). The strongest frequency in the spectrum is $f_{1}$, followed by $f_{1}-f_{2}$.

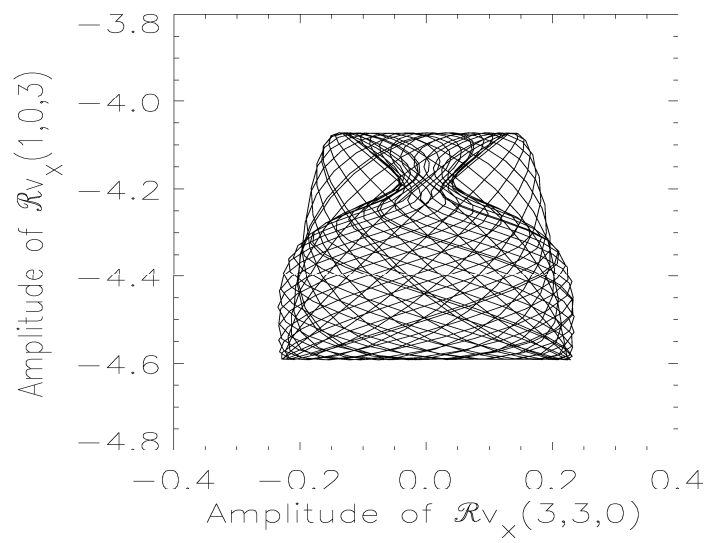

FIG. 4. Phase space trajectory projected onto the plane spanned by $\Re \tilde{v}_{x}(3,3,0)$ and $\Re \tilde{v}_{x}(1,0,3)$ at $\mathcal{R}=32000=48.7 \mathcal{R}_{c}$. 